\title{
Electronic Highways in Bilayer Graphene
}

\author{
Zhenhua Qiao $^{\dagger}{ }^{1}{ }^{\text {Jeil Jung }}{ }^{\ddagger},{ }^{1}$ Qian Niu $^{\S},{ }^{1,2}$ and Allan H. MacDonald ${ }^{1}$ \\ ${ }^{1}$ Department of Physics, The University of Texas at Austin, Austin, Texas 78712, USA \\ ${ }^{2}$ International Center for Quantum Materials, Peking University, Beijing 100871, China
}

(Dated: June 5, 2018)

\begin{abstract}
Bilayer graphene with an interlayer potential difference has an energy gap and, when the potential difference varies spatially, topologically protected one-dimensional states localized along the difference's zero-lines. When disorder is absent, electronic travel directions along zero-line trajectories are fixed by valley Hall properties. Using the Landauer-Büttiker formula and the non-equilibrium Green's function technique we demonstrate numerically that collisions between electrons traveling in opposite directions, due to either disorder or changes in path direction, are strongly suppressed. We find that extremely long mean free paths of the order of hundreds of microns can be expected in relatively clean samples. This finding suggests the possibility of designing low power nanoscale electronic devices in which transport paths are controlled by gates which alter the inter-layer potential landscape.
\end{abstract}

PACS numbers:

More than half a decade after seminal transport studies of graphene sheets demonstrated the material's half-quantized quantum Hall effect [1, 2], interest is turning toward applications of the material's exceptional properties [3, 4]. The half-quantized Hall effect in graphene is a manifestation of momentum-space Berry phases associated with its sublattice pseudospin degree-offreedom [5-9]. The physics explored in the present paper centers on one dimensional (1D) states which have the same origin as those that appear in bilayer graphene [10], monolayer graphene [11], and chirally stacked multilayer graphene [12, 13] along zero-lines of inversion-symmetry-breaking potentials. When uniform, these potentials open gaps and induce quantized but canceling quantized Hall responses from $\pi$-orbital electronic states near $K$ and $K^{\prime}$ valleys. The quantized Hall response can be calculated by integrating the momentum-space Berry curvatures over occupied valence band states [10-12], and changes sign when the inversion-symmetry-breaking potential changes sign. The 1D states we study are closely related to the edge states which are always present at spatial boundaries between regions with different quantized Hall conductances. Because the valley Hall conductivity in chirally stacked $N$ layer graphene, $\sigma_{x y}^{v}=N e^{2} / 2 h$, the number of interface channels per valley localized along a zero-line is also equal to $N$ [13]. Similarly, because opposite valleys have opposite Hall conductance sign, the chiral edge states associated with different valleys travel in different directions. Below we refer to the 1D states localized near the zero-lines as kink states.

The possibility of controlling current paths in graphene with gates has been explored previously by studying bipolar p-n
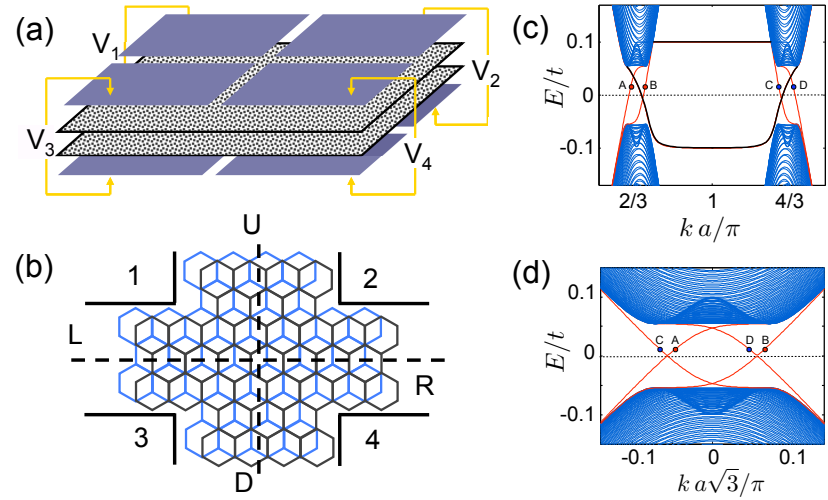

FIG. 1: (Color Online) (a) Model device with four regions that can be gated to positive or negative inter-layer potential values. (b) Schematic representation of the lattice geometry used in our numerical simulations. The horizontal and vertical axes are chosen to be aligned along the zigzag and armchair honeycomb lattice directions of both the device and the graphene bilayer reservoirs used in our four-probe NEGF calculations. (c) One-dimensional band structure of a zigzag ribbon in which the inter-layer potentials of Eq. 1 change sign at the ribbon center: $U_{i}= \pm V(x)= \pm 0.1 t \operatorname{sgn}(x)$. Two 1D modes traveling in each direction are spatially localized at the ribbon center. Right going states are labeled with letters A, B whereas left going states are labeled as C, D. Additional 1D channels appear in the gap that are localized near opposite edges of the ribbon. These states are plotted in black and are doubly degenerate due to inversion symmetry across the ribbon. (d) The band structure in the armchair case has two pairs of oppositely propagating channels located at the ribbon center but does not support edge states. The atomically scale sharp potential variation we used leads to a small avoided crossing gap $\Delta \sim 0.0014 t$ at the neutrality point. The size of this gap shrinks rapidly when the potential variation becomes smoother. 


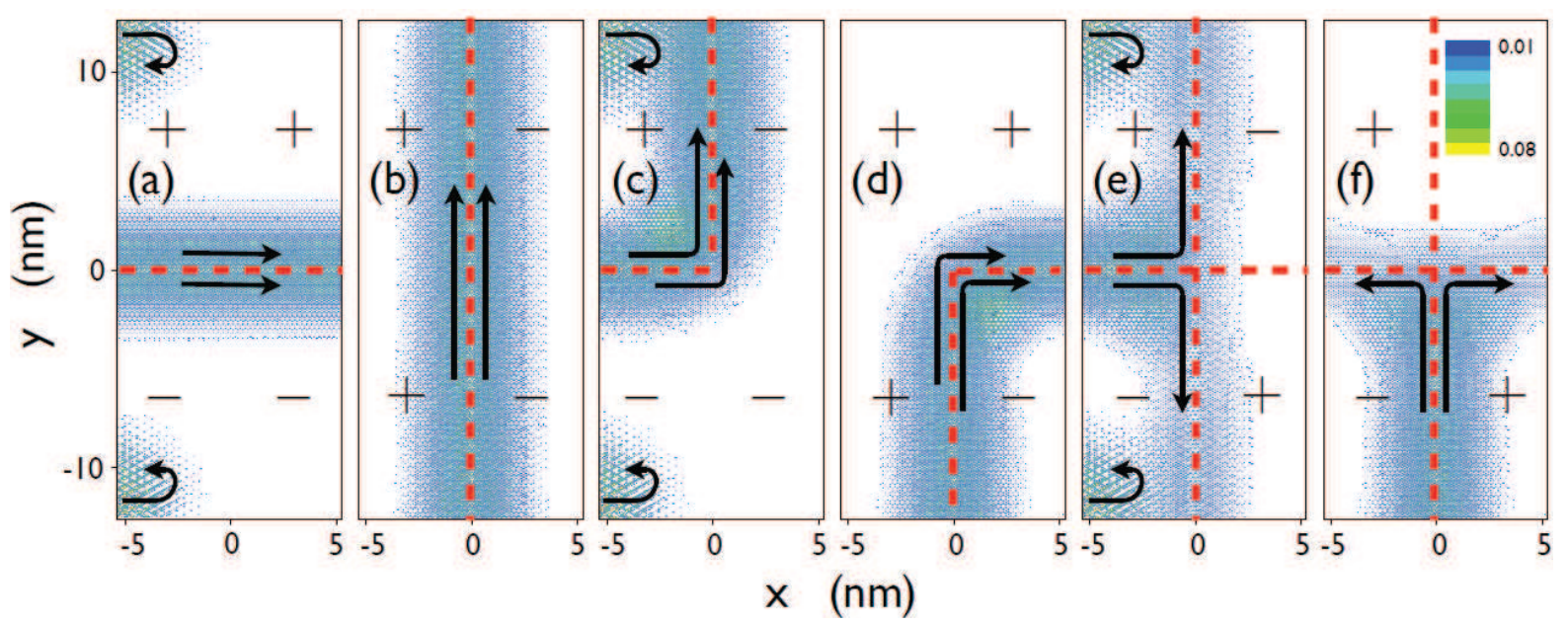

FIG. 2: (Color Online) Fermi-level kink scattering-state density distributions in four-probe bilayer graphene cross-bars for representative potential difference zero-line configurations. The left, right, up, and down reservoirs each have a single zero-line at the ribbon center which matches a system zero line at its boundary. Each point in these plots corresponds to an individual carbon atom site. The crystal is oriented so that the $x$ axis is along a zigzag direction and the $y$ axis along an armchair direction. The thick dotted lines indicate the potential difference zero-lines while the \pm symbols specify the potential difference sign in four quadrants imagined to be controlled by four separate front/back gate pairs. The panels (a), (c), and (d) are for scattering states incident from the left lead while (b), (d), and (f) are for scattering states incident from the lower lead. Transmission along an isolated zero [(a-d)] line is essentially perfect. When two zero-lines intersect [(e-f)], the current splits into contributions that follow the two available outgoing paths. In all cases chirality is preserved, even when the path bends. In cases (a-e) the low-potential region is always on the right in the direction of travel while in case (f) it is always on the left. These properties are satisfied equally well for zigzag and armchair kinks.

junctions and unipolar fibre-optic guides [14]. The present work shows that kink state conducting channels, which can be formed simply by gating bilayer graphene, are nearly ballistic in clean samples and have very long mean free paths when the disorder strength is small compared to the $2 \mathrm{D}$ bulk band gap. In addition, the kink states retain substantial valley pseudospin memory at bends and at intersections of zero-line trajectories. Our calculations strongly suggest that pseudospin electronics [5] can be realized by controlling internal zero-line trajectories. As long as a kink-state electron maintains its valley label, its direction of travel along a zero-line is uniquely determined. Transport properties are then completely determined by zero-line topology. In the case of $N=2$ bilayer graphene the symmetry breaking potential is simply the electric potential difference between layers [15], which is easily altered by gates as illustrated in 11a). This setup constitutes the simplest example of the valley valve proposal consisting of two valley filters placed sequentially. [10]

Since valley label is a good quantum number only in the absence of disorder, and then only for straight zero-lines that do not follow armchair directions, it is critical to address the robustness of valley memory. In this article we report on a numerical non-equilibrium Green's function (NEGF) study of the influence of bends and disorder on kink state transport properties. We employ a $\pi$-band tight-binding model rather than a continuum model $[10,16]$ which automatically conserves valley index. We find that current paths nevertheless follow continuum model predictions to a remarkable degree. This property still holds when portions of the zero-line follow one of the armchair directions even though armchair ribbons do not support edge states at the ribbon/vacuum boundary[13, 17].

The band structures of straight zigzag and armchair ribbons with a zero-line along the ribbon center are illustrated in panel (c) and (d) of 1 The anticipated pair of kink states localized at the sample center appears for both ribbon orientations. For the zigzag case in panel (c), kink states appear near ribbon wavevectors $k=2 \pi / 3 a, 4 \pi / 3 a$ where $a=2.46 \AA$ is the lattice constant of graphene, as suggested by a bulk graphene band projection[13]. Zigzag edges support edge state channels localized at the ribbon/vacuum edges in addition to the kink states, whereas armchairs ribbons do not support edge states and all kink states appear near 1D momentum $k=0$. The close proximity of opposite-velocity kink states in both real-space and 1D momentum space might suggest that the continuum model picture should fail badly when the zero-line direction is close to an armchair direction. We will show that this is not the case.

Kink states have definite chirality if they preserve their valley labels; states in one valley propagate along zero-lines keeping low-potential regions on the left, while states in the other valley keep low-potential regions on the right. Zero-lines intersect when the potential difference landscape has a zero saddle point. For a general continuous potential-difference profile, a system can have many zero lines some of which are closed. When valley memory is perfectly retained, only open paths connected to reservoir zero-lines are relevant for transport. Zero-line considerations are therefore relevant to the analysis of transport in neutral 
systems with smooth random potential differences. In this paper, however, we concentrate on systems with simple gate-defined potential-difference profiles designed to control current paths in bilayer graphene systems.

We model the case in which the leads are bilayer graphene ribbons with a single zero-line at their centers, and the system is divided into four quadrants in which the sign of the potential difference can be varied independently as indicated schematically in 1 a). Both incoming and outgoing states in the leads therefore have definite pseudospin labels. If pseudospin memory were perfect, injected electrons would travel following the continuation of the lead's zero-line to one of the reservoirs.

Our explicit calculations are based on the $\pi$-orbital tight-binding model:

$$
H=-\sum_{\langle i, j\rangle} \gamma_{i, j} c_{i}^{\dagger} c_{j}+\sum_{i} U_{i} c_{i}^{\dagger} c_{i}
$$

where $U_{i}$ is a $\pi$-orbital site energy and $\gamma_{i, j}$ is either a nearest-neighbor in-plane hopping amplitude with value $t=2.6 \mathrm{eV}$ or a vertical inter-layer hopping amplitude with value $t_{\perp}=0.34 \mathrm{eV}$. The trigonal warping $\gamma_{3} \sim 0.1 t$ term can play a role in the limit of vanishingly small gaps [18] but are unimportant for our present discussion, as we show in the supporting information. Here $c_{i}^{\dagger}$ and $c_{i}$ are $\pi$-orbital creation and annihilation operators for site $i$. In most of our numerical simulations, we have considered a bilayer graphene flake containing a total of $120 \times 92 \times 2=22080$ (vertical, horizontal, layer) atomic sites in the central scattering region, corresponding to a few hundreds of $\mathrm{nm}^{2}$ of flake area. We create kink states by setting $U_{i} \rightarrow \pm 0.1 t$ so that the sum of site energies in different layers is everywhere zero and the difference is $\pm 0.2 t$ in the \pm regions. The atomic scale variation of the potential difference is not physically realistic, of course, since the sharpness of its spatial profile cannot exceed the greater of the physical gate separation and the vertical distance between bilayer and gate. In the supplementary information we show that our results are not altered in any essential way as long as the distance over which the potential difference shifts between positive and negative values is smaller than $\sim 100 \mathrm{~nm}$. The potential differences open up gaps in the spectrum so that the only states at the Fermi level of a nearly neutral bilayer are ribbon edge states and kink states localized along zero-lines. We label the four semi-infinite bilayer graphene reservoirs in our NEGF calculations up (U), down (D), left (L) and right (R).

Our main numerical results, summarized in 2] were obtained for model flakes with zigzag edges in the horizontal direction and armchair edges in the vertical direction. By varying the gating potentials we can arrange to have vertical or horizontal zero lines in the system, to have a single zero line that rotates by $90^{\circ}$ between zigzag and armchair directions, or to have two such zero lines that intersect at the middle of the sample. Configurations like this, in which the zero lines of interest do not intersect with the edge of the system can be used to isolate kink state conducting channels from edge state conducting channels. We anticipate that disorder at the edges will tend to localize edge state transport. For the devices that we have in mind, increasing disorder at the edges may in fact be desirable in order to mitigate their possible role in transport.

We study how controlling the potential-difference profile can control transport properties by calculating the conductances between probes for each gating geometry. The conductance $G_{p q}$ from the $q$-th probe to the $p$-th probe can be evaluated from the Landauer-Büttiker formula:

$$
\mathrm{G}_{p q}=\left(e^{2} / h\right) \operatorname{Tr}\left[\Gamma_{p} \mathrm{G}^{r} \Gamma_{q} \mathrm{G}^{a}\right],
$$

where $\mathrm{G}^{r / a}$ are the retarded and the advanced Green functions of the central scattering regime which we evaluate following the same procedure as outlined in earlier work [20], with a small shift of the Fermi energy from neutrality $(E=0.001 t)$ to prevent the small avoided crossing gaps in the armchair direction from playing a role. All quantities are matrices with system carbon site labels. Here $\Gamma_{p}$ is the line-width function coupling the $p$-th probe to the scattering region, and can be calculated from the self-energy of the semi-infinite lead using the transfer matrix method [21]. Our results are summarized visually in 2 by plotting the local density of states contribution $\rho_{p}\left(\mathbf{r}, \varepsilon_{F}\right)$ of scatttering states injected from $p$-th probe. These are calculated using the formula [19]:

$$
\rho_{p}\left(\mathbf{r}, \varepsilon_{F}\right)=\frac{1}{2 \pi}\left[\mathrm{G}^{r} \Gamma_{p} \mathrm{G}^{a}\right]_{\mathbf{r r}} .
$$

We consider first the simplest setup, which has an isolated horizontal zero-line. Incoming electrons propagate ballistically across the system. The horizontal conductance is almost exactly two in units of $e^{2} / h$ as expected for this disorder free, straight zero-line case. For the horizontal zigzag orientation case illustrated in 2 a), we see that a pair of edge channels appears in addition to the kink states, as expected [13, 17, 22], for zigzag edge boundaries. Electrons incident upon the system in these edge channel states are reflected when they encounter the system's impenetrable gapped region. In the case of a straight vertical zero-line in 2 (b), current flows only through the kink channels because there are no edge states associated with the armchair edge terminations.

The second setup [panels (c) and (d)] supports an isolated zero-line which bends by $90^{\circ}$. Pseudospin index selective current propagation is demonstrated to be even more robust in this device geometry in which three system quadrants have the same 


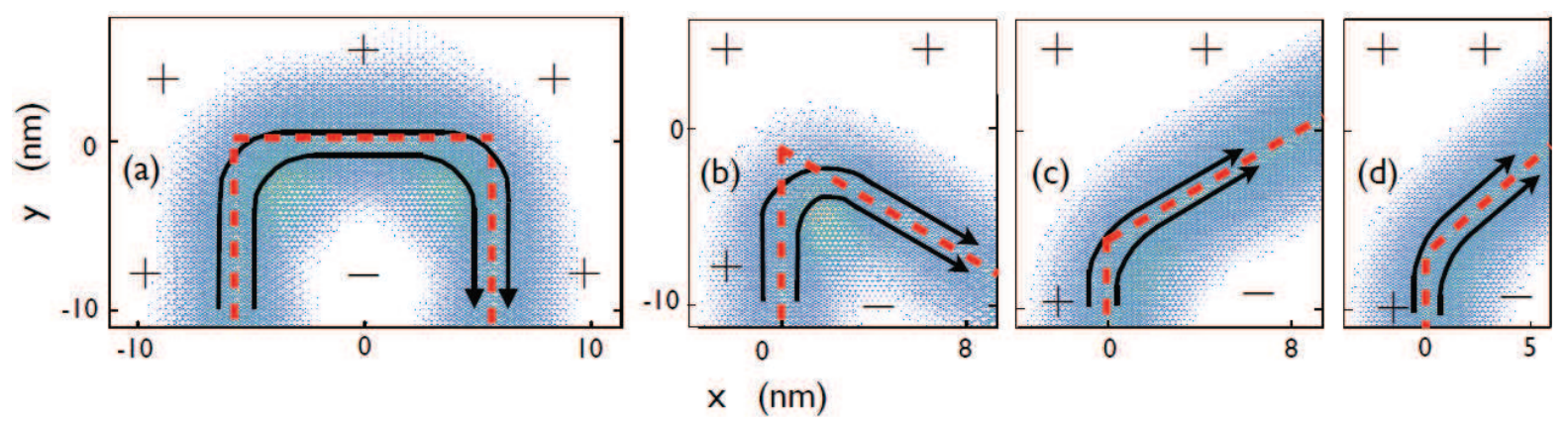

FIG. 3: (Color Online) A few examples of transport along zero lines with a variety of turn angles. In case (a) the zero line currents are reversed by consecutive $90^{\circ}$ turns changing the propagation direction first from armchair to zigzag and then from zigzag to armchair. We also show numerical results for turn angles of $60^{\circ}$ (b), and $120^{\circ}$ (c) from armchair to armchair directions. In case (d) the turn angle is $131^{\circ}$ and the final propagation path is neither zigzag nor armchair. The numerically calculated conductances between the contacts indicate almost perfect transmission along the zero-lines with no backscattering due to the finite turn angles.

difference-potential. The scattering state charge density profiles and calculated conductances show that an incoming kink current approaching the bend point makes a ninety degree turn in order to preserve its pseudospin, instead of changing its valley index and reflecting. For currents coming from the $L$ probe and making an upward turn, we obtained transmission conductances of $G_{U / R / D, L}=(1.97,0.01,0.00)$, whereas for an incoming current from the $D$ contact making a right turn the calculated conductances are $G_{L / U / R, D}=(0.01,0.00,1.97)$. The small deviation of our numerical results from the ideal transmission of two units of $e^{2} / h$ is attributed in the absence of disorder to the finite size of our simulation cell that cannot capture the entirety of the kink state wave function tails away from the domain interface. For these $90^{\circ}$ turns the kink state propagation direction changes either from zigzag to armchair or from armchair to zigag. Nevertheless there is almost no current leaking towards the other leads in the device because there are no zero-line conduction panels.

The third setup examines the alternating potential-difference profile case [see panels (e) and (f)] in which two zero-lines intersect. Our calculations show that this arrangement creates an electronic beam splitter. Propagation in the forward direction at the intersection point is forbidden by the pseudospin filtering rule since that direction of travel requires that the pseudospin be reversed. Our numerical calculations show conductances of $G_{U / R / D, L}=(0.99,0.02,0.99)$ for currents entering through the probe $L$ and $G_{L / U / R, D}=(0.99,0.00,0.99)$ for currents entering through the probe $D$, once again demonstrating almost perfect pseudospin filtering. The conspicuous absence of current density in the forward direction indicates that the valley index is well preserved beyond the path bifurcation point.

The above calculations for current densities and conductances support the existence of a well defined internal pseudospin degree of freedom that is well preserved before and after currents are forced to make turns or split at the channel bifurcation point. Absence of backscattering and conservation of pseudospin is further confirmed by calculations carried out in a six-gate geometry in which a zero line reverses direction as shown in 3 a). The pseudospin remains unchanged in which the current propagation path direction changes from armchair to zigzag and back to armchair orientations. Similarly results for current-path turn angles of $60^{\circ}$ and $120^{\circ}$ are shown in 3 (b) and (c) in which the propagation direction changes from armchair to armchair. The absence of an essential role for propagation direction is further confirmed by the case of a turn angle of $131^{\circ}$ illustrated in 3.d). In this case the current propagation direction after the turn is neither zigzag nor armchair.

Short range disorder sources, for example vacancies, grain boundaries, or other structural defects, can provide the relatively large momentum transfers necessary to backscatter kink states along non-armchair directions. We wish to the assess circumstances under which disorder can weaken the current-path control based on valley pseudospin memory discussed in the previous section. In the following we present numerical results for inter-lead transmission coefficients in the presence of short-range and long-range disorder potentials. In order to speed up these calculations we used a somewhat smaller system size than in the previous section. The results reported below are for disordered systems with $n_{y}=80$ and $n_{x}=92$ sites. Systems of this size have $N=14970$ atoms in the central scattering region.

To model short range disorder we add a random contribution to the on-site potential: $H_{d i s}=\sum_{i} \omega_{i} c_{i}^{\dagger} c_{i}$, where $\omega_{i}$ is distributed uniformly in the interval $[-W / 2, W / 2]$ with $W$ characterizing the strength of the disorder. The symmetries of our model system allow us to focus on the conductances $G_{L / R / U, D}$ and $G_{L R}$ when currents enter through probe $D$. In 4 , we show the evolution of the average conductance $\left\langle G_{p q}\right\rangle$ as a function of the disorder strength $W$ for the setups (b), (d), (f) shown in 2 . In panel (a), we see that $\left\langle G_{D U}\right\rangle$ preserves nearly perfect transmission without fluctuation for disorder strengths $W / t \in[0,0.2]$, i.e. when the disorder potential is smaller than the bulk gap in the constant potential difference regions. When the disorder strength increases further, $\left\langle G_{D U}\right\rangle$ shows a mild average decrease of around $10 \%$ and larger fluctuations between disorder realizations. For $W / t=0.8$, a 

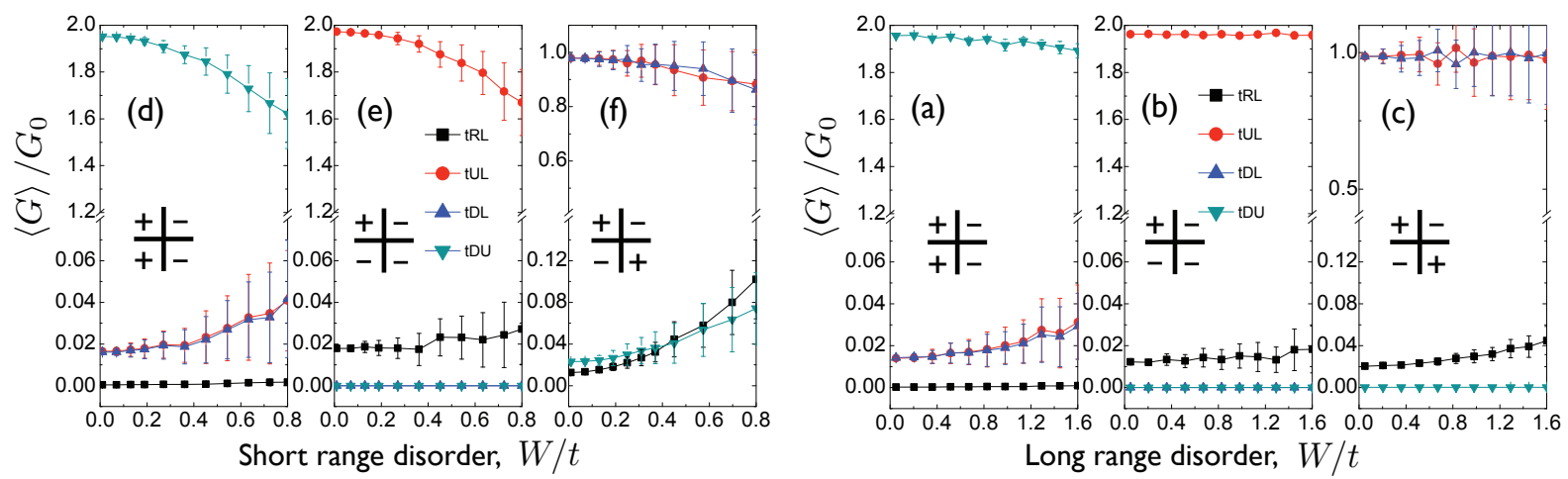

FIG. 4: (Color Online) Average conductance in units of $G_{0}=e^{2} / h$ as a function of external disorder strength for short range onsite disorder (a), (b), (c) calculated with 200 disorder realizations, and long range disorder (d), (e), (f) obtained with 100 disorder realizations. We observe that for disorder strengths comparable to or smaller than the interlayer bias, the conductances remain very close to clean-limit values. Deviations of less than $15 \%$ are seen when the disorder energy is twice the interlayer potential difference and 2/3 of the clean-limit conductivity is retained even when the disorder strength is four times larger than the interlayer potential difference. The small leakage of currents to forbidden channels shown here is largely due to the finite size of our simulation cell and is expected to be greatly reduced in larger systems. Note that we have used a different scale to represent those conductances which have small values in the disorder-free case.

disorder strength four times larger than the bulk gap, $\left\langle G_{D U}\right\rangle$ can still reach $75 \%$ of its original conductance. The small leakages of current along the forbidden directions at the bottom of the figure which appear even in the clean limit reflects insufficient system size to completely eliminate these tunneling transmission paths. This quantity is however very small and its further increase due to scattering introduced by disorder does not surpass $2 \%$ of the total conductance for the strongest disorder strengths we have considered.

Similar trends are seen in the other setups examined in panels (b) and (c), confirming that ballistic transport due to valley pseudospin memory is extremely robust for disorder strengths smaller than or comparable to the system gap.

We have also considered disorder models with finite range correlations of the disorder potential by examining the model $V_{i}=\sum_{j} \omega_{j} \exp \left(-\left|\mathbf{r}_{j}-\mathbf{r}_{i}\right|^{2} / 2 \xi^{2}\right)$ where we used $\xi=5 a$ in the present calculations. The sum is carried out over all the neighboring sites and the local disorder strength $\omega_{j}$ is bracketed between the interval $[-\widetilde{W} / 2, \widetilde{W} / 2]$. The renormalization of disorder strength by $\widetilde{W}=W /(2 \xi / a)^{2}$ as a function of $\xi$ allows a more consistent comparison with the local disorder model and reflects the relative increase of disorder strength due to the long range. The above relation was found through an empirical fitting to data obtained summing the effects of the Coulomb potential generated by all the surrounding long range impurity sites with constant strength $W$. The factor 4 is slightly smaller than a value of $2 \pi$ that we would obtain analytically from an integration in 2D space. Our numerical results for this model are illustrated in panels (d)-(f) of 4 , where we plot the average conductance $\langle G\rangle$ as a function of the effective disorder strength $W$. The conductivities we obtained for long range disorder show an even greater robustness than the results obtained with short range disorder potentials.

To identify the physics behind the robustness of kink states against disorder, we have calculated the local density of states as it propagates along the disordered medium and illustrated our findings in 5 From the calculated local density-of-states we notice that even for moderately strong disorder comparable with the band gap, as shown in panel (b), the transverse profile of the kink states is not modified significantly during transmission. (There is a decrease in the magnitude of the local density-of-states upon moving along the zero-line transmission path due to back scattering.) This robustness of the kink channels to disorder also explains why the conductance along the forbidden channels represented in 4 hardly increases with disorder strength. It is only in the limit of very strong disorder as shown in panel (c), that the 1D character of the kink channels is completely destroyed, spreading the wave function more homogeneously across the ribbon. In panel (d) we have plotted the evolution of the averaged conductivity $\langle G\rangle$ as a function of position obtained by evaluating recursively the resolvent between the first and the $N$-th unit cell layer in an armchair ribbon. These results demonstrate that the backscattering rate is fairly small as long as the kink state retains its original shape.

Further insight into the suppression of backscattering in kink state transport in the weak disorder limit from an initial kink state $i$ to one of the two final kink states $f$ with opposite velocities can be gained from the mean free path calculation of an incident state $i$ using the Fermi's Golden-Rule approximation. These 1D kink states conducting channels are highlighted by dots in the corresponding band structures in $1(\mathrm{c})$ and (d), where we have designated the right-going states by labels A, B and the left goers by labels $\mathrm{C}, \mathrm{D}$. The transverse eigenvectors of these states are described by coefficients $c_{i, \tau}$ and $c_{f, \tau}$ where $i=\mathrm{A}, \mathrm{B}$ and $f=\mathrm{C}, \mathrm{D}$, and $\tau$ labels lattice sites across the ribbon. With this notation we can measure the degree to which channels overlap 

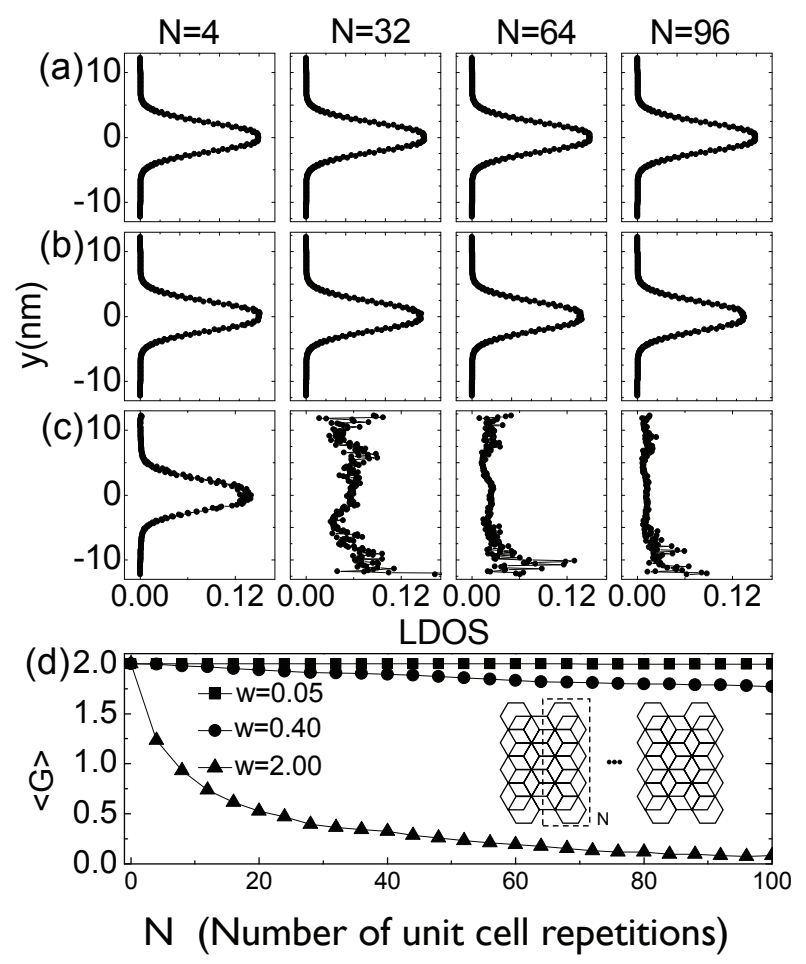

FIG. 5: Transverse local density-of-states profiles for at a series of positions in the scattering region at a series of points along the zero-line transmission path for a wide armchair ribbon with a bulk gap $E_{g} \sim 0.1 t$ and short range disorder strengths of $W / t=0.05,0.4,2$ in panels (a), (b) and (c) respectively. The kink states are spread over a width of about $L \sim 10 \mathrm{~nm}$ and are not easily backscattered even for disorder strengths comparable to the band-gap, as shown in panel (d). In the limit of very strong disorders we can observe the spreading of the kink state wave functions throughout the whole ribbon and a substantial decrease of the conductivity $G$ as it propagates.

spatially by defining $S_{i f}=\sum_{\tau}\left|c_{i, \tau}\right|^{2}\left|c_{f, \tau}\right|^{2}$. The Golden rule decay time of a state $|k n\rangle$ into a set of final states $\left|k^{\prime} n^{\prime}\right\rangle$ can be written as

$$
\tau_{k, n}^{-1}=\frac{2 \pi}{\hbar} \sum_{k^{\prime} n^{\prime}}\left|\left\langle k^{\prime} n^{\prime}|V| k n\right\rangle\right|^{2} \delta\left(\varepsilon_{k^{\prime} n^{\prime}}-\varepsilon_{k n}\right) .
$$

The mean free path is related with the inverse of the decay time (in units of the unit cell length along the ribbon $\widetilde{a}$ ) and is given by (we refer the reader to the supporting information for more details of the derivation)

$$
\frac{l_{i}}{\widetilde{a}}=\frac{v_{i} \tau_{i}}{\widetilde{a}}=\frac{12 \hbar^{2}}{\widetilde{a}^{2} W^{2}} \frac{v_{i}}{\sum_{f} S_{i f} / v_{f}}=\frac{C_{i}}{W^{2}} \sim \frac{6 E_{g}^{2} M}{W^{2}}
$$

where we used the relation $\overline{\omega^{2}}=W^{2} / 12$ for uniformly distributed disorder strength $\omega$ within an interval $W$. The rightmost approximate expression was found using $\hbar\left|v_{i, f}\right| \sim E_{g} \widetilde{a}$ where $E_{g}$ is the bulk band gap and $S_{i f} \sim 1 / M$. The dimensionless number $M \sim 290$ for bilayer armchair ribbon kink states indicates the effective number of transverse lattice sites over which the transverse density is significant. This number is typically of the order a few hundred and is expected to become larger when the kink potential becomes smoother since it is essentially proportional to the real space width of the kink state. The coefficients in Eq. 4 evaluated for scattering of the kink states indicated in 1 are $C_{A} / t^{2}=2.3 \times 10^{3}$ and $C_{B} / t^{2}=1.5 \times 10^{3}$ for armchair directions. This argument suggests that the mean free path of a kink channel can be of the the order of micrometers even when the disorder strength is comparable with the bulk band gap, and that it will increase quadratically for weaker disorder.

The reason behind the suppression of backscattering for short range disorder is the spreading of kink states over several hundred carbon atoms, which makes it difficult for an isolated scatterer to globally modify a given kink state and reverse its direction. Backscattering for smooth long range disorder is even more efficiently suppressed due to essentially perfect orthogonality between transverse eigenvectors in different 1D channels. (As discussed in the supplementary information the pair of $\mathrm{AB}$ and $\mathrm{CD}$ kink states when they propagate along the zigzag direction have symmetric and antisymmetric eigenvector amplitudes manifested in units of two carbon lattices. The $\pm k$ transverse states for armchair channels are complex conjugates and mutually orthogonal.) 
The mechanism for the suppression of backscattering of kink states is different from that relevant to the quantum Hall effect in which counter propagating sates are spatially separated [24], and different from that relevant to the quantum spin Hall effect in which counter-propagating states do overlap spatially but are decoupled when time-reversal invariance is present [25], but closely related to the mechanism that supresses backscattering in large-diameter metallic carbon nanotubes [26]. This mechanism is unlikely to lead to perfectly quantized transport in large systems, but is nevertheless quite effective and can lead to extremely long mean free paths of the order of hundreds of microns in relatively clean samples.

In summary, an interlayer potential difference in bilayer graphene can open up a band gap that can be as large as $\sim 0.3$ $\mathrm{eV}$ [27]. One interesting feature of the electronic structure of these electrically tunable semiconductors is the presence of large Berry curvature peaks of opposite sign sharply localized near the two Dirac points of the material. The Berry curvature is associated with the momentum dependence of the Bloch state sublattice content, i.e. with the sublattice pseudospin. When momentum space is separated into regions centered on the two valleys these Berry curvatures suggest the presence of nearly perfectly quantized Hall effects of opposite sign associated with the two valleys, i.e. they suggest a valley Hall effect. In this paper we have reported on a numerical study bilayer graphene ribbons in which the inter-layer potential, and hence the Hall conductances, change sign as a function of position. Our study focuses on the chiral edge state channels [10] localized along zero-lines of the inter-layer potential that are associated with the valley Hall effect. As long as valley label is preserved the chiral states provide one-way current transport channels which can be manipulated by modulating the inter-layer potential profile, and in particular the paths of its zero-lines.

Our numerical study examines the robustness of these one-way kink-state transport channels when the transport channel bends changing the bilayer graphene crystal trajectory. This point requires the use of a microscopic lattice model and not a continuum model [10] in which valley identity is automatically retained. Since the kink states are associated mainly with small well separated regions of momentum space they are expected to approximate the chiral perfect transmission properties associated with the quantum Hall effect. Our numerical study shows that backscattering from a kink state associated with one valley to a kink state associated with the other valley is small independent of bend angles in the zero-line path. This property continues to hold even when portions of the zero-line path follow armchair directions, along which the two valleys have identical 1D momentum projections [13]. The small bend resistance values differ starkly from the case of ordinary semiconductor quantum wires [28, 29] in which envelopes satisfy non-relativistic wave equations. We have found essentially zero bend resistance due to a perfect transmission along zero lines despite the sharp turns in the current propagation path, unlike in electron waveguides formed from GaAs-AlGaAs wafers.

We have also demonstrated that one-way conductance through kink channels is extraordinarily robust against both short-range and long-range disorder potentials. We have attributed this behavior to long kink-channel mean-free-paths in the weak disorder limit. A Fermi golden-rule analysis, in which the the mean-free-path depends quadratically on the ratio between the bulk band gap size and disorder strength, suggests that kink-channel mean-free-path values from tens to hundreds of microns should be achievable in bilayer graphene samples of typical mobility. The robustness against even short range disorder is essentially due to the wide several nanometer lateral spread of the kink state wave functions which reduces the effectiveness of lattice scale disorder. This behavior is analogous to the familiar inverse diameter dependence of the backscattering probability in metallic carbon nanotubes [26].

Graphene samples obtained through mechanical exfoliation have relatively few short range disorder defects in the bulk. We therefore expect that efficient pseudospin selective beam splitters and current direction switches can be manufactured based on bilayer graphene samples accesible by current experimental methods. Such a possibility would open avenues for exploring new $1 \mathrm{D}$ transport physics in an experimentally controllable manner.

We acknowledge financial support received from Welch Foundation grants F-1255 and TBF1473, NRI-SWAN, DOE grant Division of Materials Sciences and Engineering DE-FG03-02ER45958, and NSF (DMR0906025). The Computer Center of The University of Hong Kong is gratefully acknowledged for high-performance computing assistance [supported in part by a Hong Kong UGC Special Equipment Grant (SEG HKU09)].

${ }^{\dagger}$ zhqiao@physics.utexas.edu;

*jeil@physics.utexas.edu;

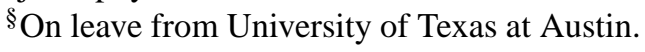

[1] Novoselov K. S. et al., Nature 438, 197 (2005).

[2] Zhang Y. , Tan Y.-W., Stormer H.L., and Kim P., Nature 438, 201 (2005).

[3] Geim A. K. and MacDonald A. H., Phys. Today 60(8), 35 (2007).

[4] Castro Neto A. H., Guinea F., Peres N. M. R., Novoselov K. S., and Geim A. K., Rev. Mod. Phys. 81, 109162 (2009).

[5] Rycerz A., Tworzydlo J. and Beenakker C. W. J., Nature Physics 3, 172 (2007).

[6] Akhmerov A. R., Bardarson J. H., Rycerz A., and Beenakker C. W. J., Phys. Rev. B 77, 205416 (2008). 
[7] Abedinpour S. H. et al., Phys. Rev. Lett. 99, 206802 (2007).

[8] Oostinga J.B. et al., Nature Mat. 7, 151 (2008).

[9] San-Jose P., Prada E., McCann E., and Schomerus H., Phys. Rev. Lett. 102, 247204 (2009).

[10] Martin I., Blanter Ya. M., and Morpurgo A. F., Phys. Rev. Lett. 100, 036804 (2008).

[11] Yao W., Yang S. A. and Niu Q., Phys. Rev. Lett. 102, 096801 (2009).

[12] Zhang F., Jung J., Fiete G. A., Niu Q. and MacDonald A. H., Phys. Rev. Lett. 106, 156801, (2011).

[13] Jung J., Zhang F., Qiao Z. and MacDonald A. H., arXiv:1105.3666 (2011).

[14] Williams J. R., Low T., Lundstrom M. S. and Marcus C. M., Nature Nanotech., DOI: 10.1038/NNANO.2011.3.

[15] McCann E., Phys. Rev. B 74, 161403(R) (2006).

[16] Killi M., Wei T.-C., Affleck I., and Paramekanti A., Phys. Rev. Lett. 104, 216406 (2010).

[17] Li J., Martin I., Buttiker M., Morpurgo A. F., Nature Physics 7, 38 (2011).

[18] Núñez A. S., Suárez Morell E., and Vargas P., arXiv: 1012.4318 (2010).

[19] Datta S., 'Electronic Transport in Mesoscopic Systems', Cambridge University Press (1995).

[20] Qiao Z., Wang J., Wei Y., and Guo H., Phys. Rev. Lett. 101, 016804 (2008).

[21] Lopez Sancho M. P., Lopez Sancho J. M., Sancho J. M. L. and Rubio J., J. Phys. F: Met. Phys. 15, 851 (1985).

[22] Castro E. V. et al., Phys. Rev. Lett. 99, 216802 (2007).

[23] Wakabayashi K., Takane Y., Yamamoto M., and Sigrist M., New J. Phys., 11, 095016 (2009).

[24] Büttiker M., Phys. Rev. B 38, 9375 (1988).

[25] Kane C. L., Mele E. J., Phys. Rev. Lett. 95, 146802 (2005).

[26] White C. T. and Todorov T. N., Nature 393, 240 (1998).

[27] Zhang Y. et al., Nature 459, 820 (2009).

[28] Takagaki Y. et al., Solid State Commun. 68, 1051 (1988).

[29] Timp G. et al., Phys. Rev. Lett. 60, 2081 (1988). 